\title{
LETTER
}

\section{Cladding-pumped Raman fiber laser with $0.78 \%$ quantum defect enabled by phosphorus-doped fiber}

\author{
Xiaoya Ma, Jiangming Xu, Jun Ye, Yang Zhang, Liangjin Huang, Tianfu Yao, Jinyong Leng, Zhiyong Pan, \\ and $\mathrm{Pu}$ Zhou \\ College of Advanced Interdisciplinary Studies, National University of Defense Technology, Changsha 410073, China \\ (Received 29 August 2021; revised 19 December 2021; accepted 27 December 2021)
}

\begin{abstract}
The quantum defect (QD) is an important issue that demands prompt attention in high-power fiber lasers. A large QD may aggravate the thermal load in the laser, which would impact the frequency, amplitude noise and mode stability, and threaten the security of the high-power laser system. Here, we propose and demonstrate a cladding-pumped Raman fiber laser (RFL) with QD of less than 1\%. Using the Raman gain of the boson peak in a phosphorus-doped fiber to enable the cladding pump, the QD is reduced to as low as $0.78 \%$ with a $23.7 \mathrm{~W}$ output power. To our knowledge, this is the lowest QD ever reported in a cladding-pumped RFL. Furthermore, the output power can be scaled to $47.7 \mathrm{~W}$ with a QD of $1.29 \%$. This work not only offers a preliminary platform for the realization of high-power low-QD fiber lasers, but also proves the great potential of low-QD fiber lasers in power scaling.
\end{abstract}

Keywords: cladding pumping; low quantum defect; phosphorus-doped fiber; Raman fiber laser

\section{Introduction}

Owing to their compact structure, high conversion efficiency and robust operation, high-power fiber lasers have attracted much research interest and have been widely employed in many fields ${ }^{[1-4]}$. Rare-earth-doped fiber lasers, as a significant mainstay of high-power fiber lasers ${ }^{[5-9]}$, however, are challenged by waste heat accumulation in the active fiber ${ }^{10]}$, especially with the power increasing beyond kilowatt level ${ }^{[11-15]}$. The quantum defect (QD) is one of the most important heat sources in the gain fiber, which could result in serious thermal effects on fiber lasers, such as the thermal lens effect, thermal mode instability and additional noise ${ }^{[16-18]}$.

Continuous efforts have been focused on reducing the $\mathrm{QD}^{[19-23]}$. Benefiting from fast development of the highbrightness pump source, Raman fiber lasers (RFLs) have been rapidly developed and widely investigated in recent years ${ }^{[24-27]}$. Surprisingly, fiber lasers based on stimulated Raman scattering (SRS) show outstanding performance for thermal management at the $1 \mu \mathrm{m}$ band. Generally, the QD

Correspondence to: J. $\mathrm{Xu}$ and $\mathrm{P}$. Zhou, College of Advanced Interdisciplinary Studies, National University of Defense Technology, Changsha 410073, China. Email: jmxu1988@163.com (J. Xu); zhoupu203@163.com (P. Zhou) of RFLs is around $5 \%{ }^{[27]}$, which is half that of the common laser diode (LD)-pumped $\mathrm{Yb}$-doped fiber laser. Employing tens of meters or even km-level passive fiber, RFLs can effectively relieve the density of the thermal deposition. Besides, the characteristic of non-photon-darkening enables RFLs to maintain stable operation. Accordingly, the RFL is a promising alternative for both high-power and low-QD fiber lasers. Up to hundreds of watt-level high-power RFLs in allfiber formats have been demonstrated by several independent groups ${ }^{[28-31]}$. By adopting a main-oscillator-power-amplifier structure, up to $3 \mathrm{~kW}$ RFLs have been demonstrated ${ }^{[32,33]}$. Usually, the high-power fiber lasers aforementioned have QDs of about $4 \%-5 \%$ based on the $13.2 \mathrm{THz}$ frequency shift. Note that a challenge in power scaling of RFLs has also been revealed and observed very recently ${ }^{[34-37]}$, where further decreasing the QD of the RFL is one of the promising solutions for power scaling.

Moreover, it has been reported that there is a gain peak with a frequency shift of less than $4 \mathrm{THz}$, called the boson peak in phosphorus-doped fiber (PDF), which may enable RFLs to further reduce the $\mathrm{QD}^{[38]}$. Recently, up to 100 -wattlevel core-pumped RFLs with less than 1\% QD have been demonstrated ${ }^{[39]}$. The results have indicated that the RFL gained by the boson peak of the PDF has the ability to realize low-QD output. Nevertheless, it is difficult to enhance 
the pump density for power scaling in conventional corepumped RFLs due to the limitations of the power handling capacity and the small mode area of the core in a singlemode fiber. One of the solutions to this challenge is cladding pumping ${ }^{[2,5,8,40-44]}$. The pump light is guided in the cladding of the fiber, which can significantly decrease the requirement on the pump brightness. Since the first demonstration of the cladding-pumped Raman regime ${ }^{[45]}$, there has been growing research interest, and significant advances in high-power RFLs have been successfully achieved ${ }^{[46-48]}$. However, for thermal management, the QDs of cladding-pumped RFLs currently reported were almost more than $4 \%$, and the realization of a further reduced QD has been dilatory owing to weak gain intensity with a tiny frequency shift of passive fiber and low pump intensity in the fiber cladding, which follows the intense gain competition and eliminates the short signal wavelength. Should the QD of the claddingpumped RFL be further decreased, it would be a promising solution for high-power fiber lasers because it combines the advantages of RFLs, cladding pumping and low heat production.

In this paper, we demonstrate a cladding-pumped RFL with QD reduced to less than 1\%, which is the lowest QD ever achieved in a cladding-pumped RFL, to our knowledge. Gained by the boson peak of the PDF, $0.78 \%$ QD with output power of $23.7 \mathrm{~W}$ has been successfully achieved. Furthermore, by optimizing the system parameters, such as the output coupler (OC) reflectivity and the fiber length, the Raman power can be scaled up to $47.7 \mathrm{~W}$ with a QD of $1.29 \%$. This work experimentally verifies the feasibility and potential of low-QD RFL.

\section{Experimental setup}

The experimental setup is a forward-pumped Raman oscillator, as shown in Figure 1(a). A home-made amplified spontaneous emission (ASE) source is used as the pump, with a wavelength tuning range of 1050-1080 $\mathrm{nm}$ and 3$\mathrm{dB}$ bandwidth of $1.7 \mathrm{~nm}^{[49]}$. The usage of ASE as the pump source instead of the classical fiber-cavity-based oscillator has the advantages of low coherence and high temporal stability, which could reduce the intensity noise and improve the laser performances ${ }^{[50-52]}$. A fiber circulator with core diameter of $20 \mu \mathrm{m}$ is mounted after the ASE source, to protect the pump from the potential backward laser and to monitor the backward light from the following oscillator. Port 2 of the circulator is spliced with a Raman oscillator, which is constructed with a highly reflective fiber Bragg grating (HR FBG), a section of PDF and an OC. The HR FBG with approximately $99 \%$ reflectivity and the OC are both located at $1086.5 \mathrm{~nm}$, and are written on the used PDF. All free ends are $8^{\circ}$-cleaved to suppress the parasitic reflection.

The PDF is a piece of triple-cladding fiber with core/inner cladding diameter of $10 / 20 \mu \mathrm{m}$ and numerical apertures (NAs) of 0.07 and 0.14, respectively, as shown in Figure 1(b). Figure 2 describes the schematics of laser transmission in cladding pumping and core pumping. Compared with the core pumping scheme in Figure 2(b), the pump light in the cladding pumping scheme is coupled into the inner cladding of the gain fiber, as depicted in Figure 2(a). Thus, the refractive index from the core to the outer cladding is designed to be decreased gradually, in order to ensure the

(a) ASE Source
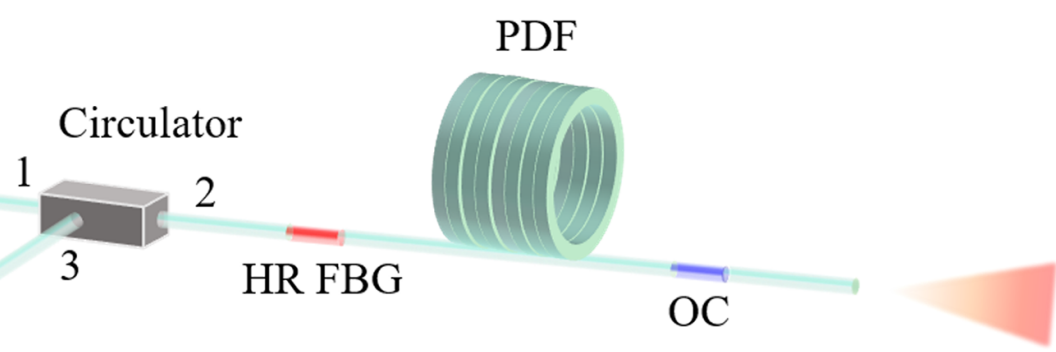

(b)

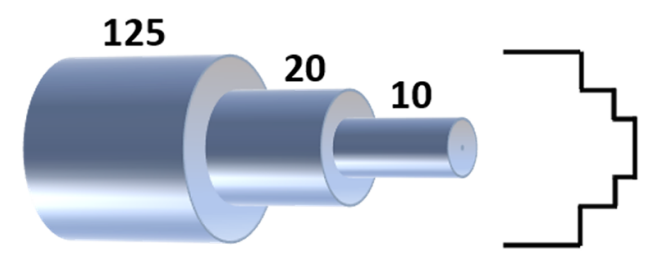

(c)

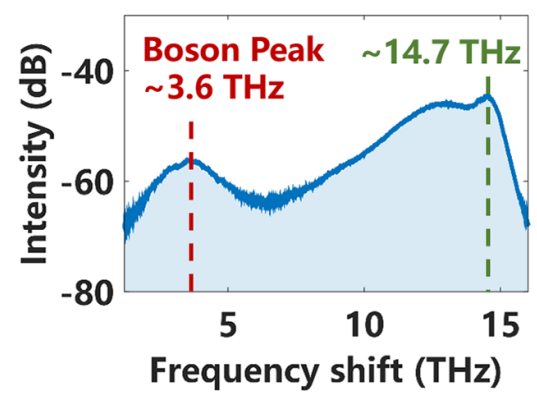

Figure 1. (a) The experimental setup of the low-QD RFL. (b) The structure of the PDF. (c) Properties of the Raman frequency shift of the PDF. 
(a) Cladding Pump

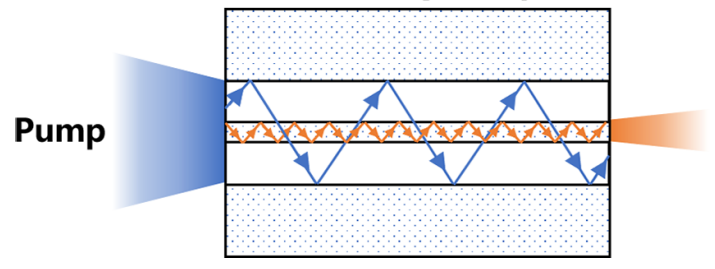

(b) Core Pump

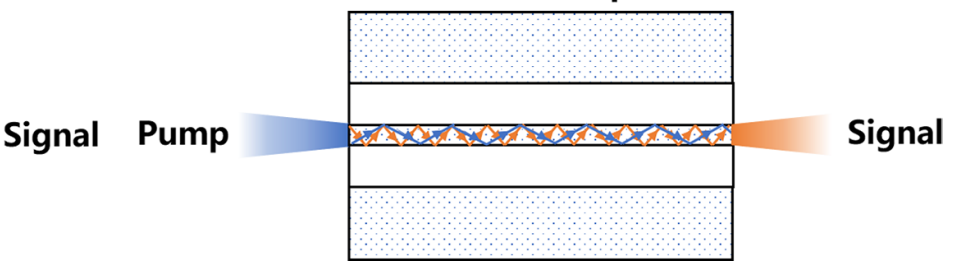

Figure 2. Schematic diagrams of laser transmission in (a) cladding pumping and (b) core pumping.

(a)

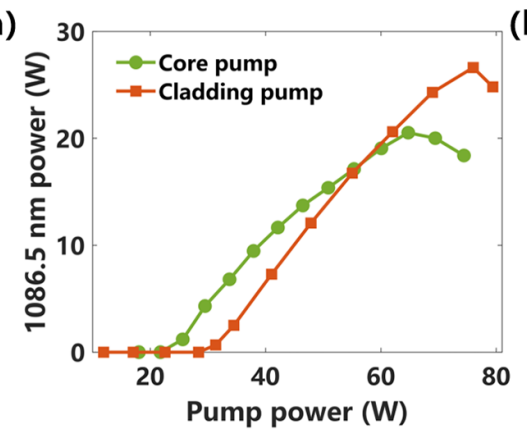

(b)

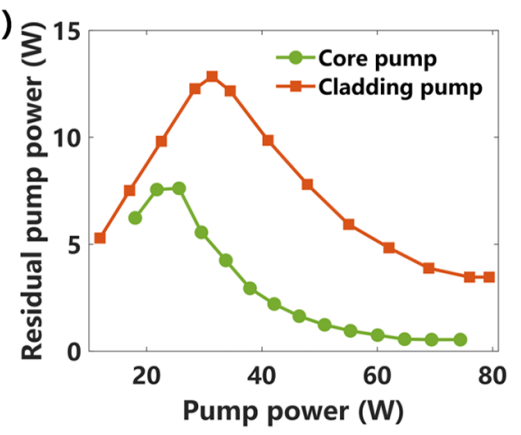

(c)

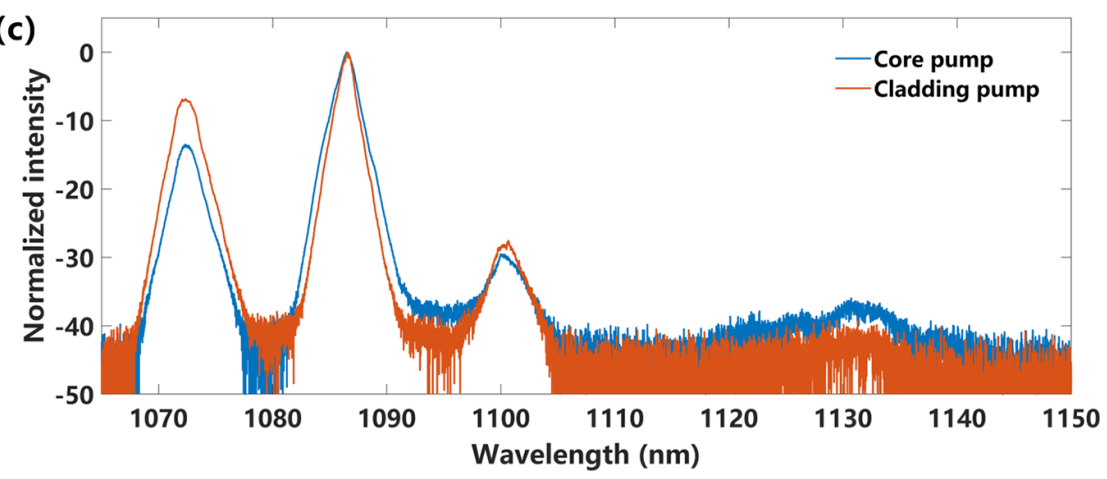

Figure 3. Power evolutions of (a) the $1086.5 \mathrm{~nm}$ signal and (b) the residual pump, in two pump schemes. (c) The normalized intensity spectra as a function of wavelength, with $55 \mathrm{~W}$ pump power.

total reflection inside the fiber. Properties of the Raman frequency shift in the range of $0-15 \mathrm{THz}$ of the PDF are shown in Figure 1(c). The frequency shift of the boson peak is located at approximately $3.6 \mathrm{THz}$. It should be noted that the phosphorus only resided in the fiber core, which enables the pump light to be converted into the signal in the core through the small frequency shift of the boson peak, as the pump propagates along the fiber.

To analyze the output properties of the cladding-pumped RFL, a core-pumped RFL using the same experimental structure is constructed as a comparison. The circulator in the core-pumped RFL has the maximal optical power from port 1 to port 2 of $100 \mathrm{~W}$, and the core/cladding diameter of the optical fiber is $10 / 125 \mu \mathrm{m}$.

\section{Results and discussion}

\subsection{Verification of cladding-pumped low-QD operation}

As the operation wavelength of the FBG pair is located at $1086.5 \mathrm{~nm}$, we firstly tuned the central wavelength of the
ASE source to $1072.5 \mathrm{~nm}$ to match the boson peak with a Raman frequency shift of approximately $3.6 \mathrm{THz}$, corresponding to a QD of $1.29 \%$. In this section, we realized the cladding-pumped low-QD RFL and contrasted the properties of cladding pumping and core pumping.

The output characteristics under the two pump schemes are analyzed, with a $1 \mathrm{~km}$ PDF and OC with $10 \%$ reflectivity, as shown in Figure 3. Figure 3(a) shows that the power of the $1086.5 \mathrm{~nm}$ signal laser depends on the pump power of the two pump schemes. It can be measured that in core pumping scheme, the lasing threshold is $21.8 \mathrm{~W}$ and the maximal signal power is $20.5 \mathrm{~W}$ at a pump power of $64.7 \mathrm{~W}$, with a slope efficiency of $47.8 \%$. Yet, in the cladding pumping scheme, due to the larger area of inner cladding, the power density of the pump with the same pump power is lower, so that the Raman laser threshold is higher than that in the core pumping scheme. In addition, with $76.0 \mathrm{~W}$ pump power, the maximal power of the $1086.5 \mathrm{~nm}$ Raman laser obtained from cladding pumping scheme reaches $26.6 \mathrm{~W}$, with a slope efficiency of $55.9 \%$. However, the residual pump power in 
the cladding pumping scheme is higher than that in core pumping scheme with the same pump power, as shown in Figure 3(b). This could be attributed to the standard circle shape of the inner cladding inside the used PDF, which cannot suppress the spiral light propagating along the fiber and can cause incomplete conversion of the pump power in the cladding ${ }^{[53]}$.

Figure 3(c) depicts the normalized intensity spectra of the two pump schemes with $55 \mathrm{~W}$ pump power. It can be found that with the same pump power, the spectral broadening of the $1086.5 \mathrm{~nm}$ peak in the cladding pumping scheme is weaker, which is caused by different distributions of the signal propagating along the fiber core between the two schemes. Under the same system condition, the pump power in the cladding pumping scheme is converted closer to the end of the fiber than that in the core pumping scheme, which can suppress the spectral broadening to some extent during the laser propagation ${ }^{[54]}$. Meanwhile, the signal light in the cladding pumping scheme can propagate a shorter distance before emitting from the fiber, causing the lower propagation loss and higher slope efficiency (see Figure 3(a)). In the cladding pumping scheme, the $1100.5 \mathrm{~nm}$ peak, which is caused by the Raman-assisted three-wave mixing (RATWM) effect $^{[55]}$, is $6.7 \mathrm{~dB}$ higher than that in the core pumping scheme. This is because there is more residual pump power in the cladding pumping scheme, which contributes to a stronger three-wave mixing (TWM) effect. The Stokes light of $1132 \mathrm{~nm}$ in the core pumping scheme is more conspicuous, and is converted from the pump wavelength through 14.7 $\mathrm{THz}$ silicon-based Raman gain. In the core pumping scheme, the pump laser, which propagates inside the fiber core, has higher power density than that in the cladding pumping scheme, which imposes stronger gain on the Stokes light of $1132 \mathrm{~nm}$ and limits the power scaling of the signal (see Figure 3(a)).

\subsection{Performance exploration of cladding-pumped low- $Q D$ RFL}

In this section, we optimized the parameters of the cladding-pumped RFL with a QD of $1.29 \%$ to improve the performance, and further explored the potential of the RFL on QD of less than $1 \%$.

To facilitate the consumption of pump power in the cladding pumping scheme, OCs with 4\%, 10\% and 19\% reflectivity are spliced with the output end of a $1 \mathrm{~km}$ PDF, respectively. The corresponding output properties of the cladding-pumped RFL are displayed in Figure 4. As the OC reflectivity increases, the feedback of the $1086.5 \mathrm{~nm}$ Raman laser is enhanced, which leads to a lower generation threshold and higher output power with the same pump power, as shown in Figure 4(a). With 19\% OC reflectivity, after the pump power exceeds the laser threshold of $22.6 \mathrm{~W}$, the power of the $1086.5 \mathrm{~nm}$ signal laser rises rapidly and reaches the maximum of $28.4 \mathrm{~W}$ with $72.5 \mathrm{~W}$ pump power. The corresponding slope efficiency is $56.9 \%$. Figure 4(b) depicts the residual pump power evolutions with different $\mathrm{OC}$ reflectivities. With an increase in the OC reflectivity, the power density of the signal laser is more intense, which causes the pump power to be converted more substantially. With $19 \%$ OC reflectivity, the residual pump power increases to $10.5 \mathrm{~W}$ and then falls off until $2.1 \mathrm{~W}$. Limited by the experimental conditions, low-QD performances with higher OC reflectivity cannot be investigated presently. If the $\mathrm{OC}$ reflectivity increases further, the high signal power density may enhance the siliconbased Raman gain and promote the generation of $1132 \mathrm{~nm}$ Stokes light, which may limit the power scaling of the signal.

To further improve the performances of the claddingpumped RFL, PDFs at lengths of $300 \mathrm{~m}, 400 \mathrm{~m}, 500 \mathrm{~m}$, $600 \mathrm{~m}$ and $1 \mathrm{~km}$ with $19 \%$ OC reflectivity are used in the Raman oscillator, respectively. The performances are compared in Figure 5. The power of the $1086.5 \mathrm{~nm}$ signal laser as a function of pump power is depicted in Figure 5(a). It can be found that the longer the fiber, the lower the laser threshold. With $400 \mathrm{~m}$ PDF, the final power of the $1086.5 \mathrm{~nm}$ signal achieves the highest value of $47.7 \mathrm{~W}$ with the maximal pump power of $89.2 \mathrm{~W}$, and the corresponding slope efficiency is $87.2 \%$. Figure 5 (b) describes the residual pump power evolutions related with the fiber length. Under the same pump power, with longer fiber, the pump power is consumed
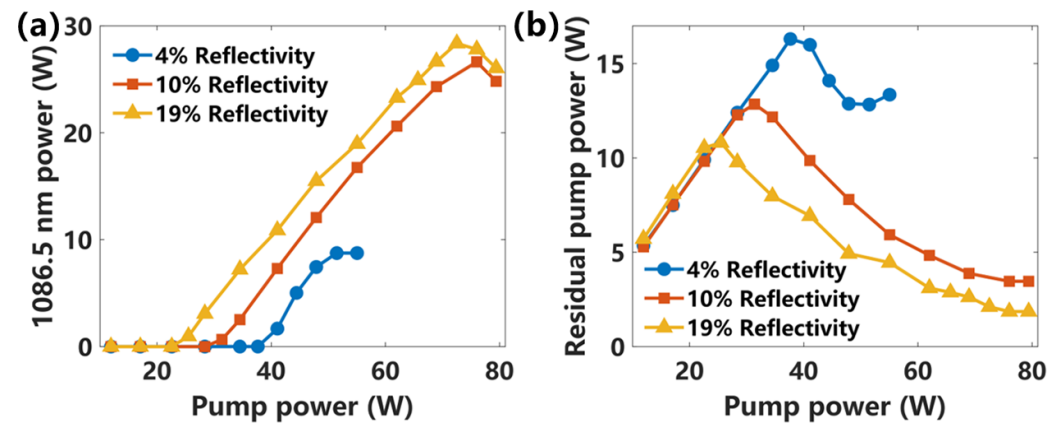

Figure 4. Power evolutions of (a) the $1086.5 \mathrm{~nm}$ signal and (b) the residual pump as functions of pump power, with different OC reflectivities. 

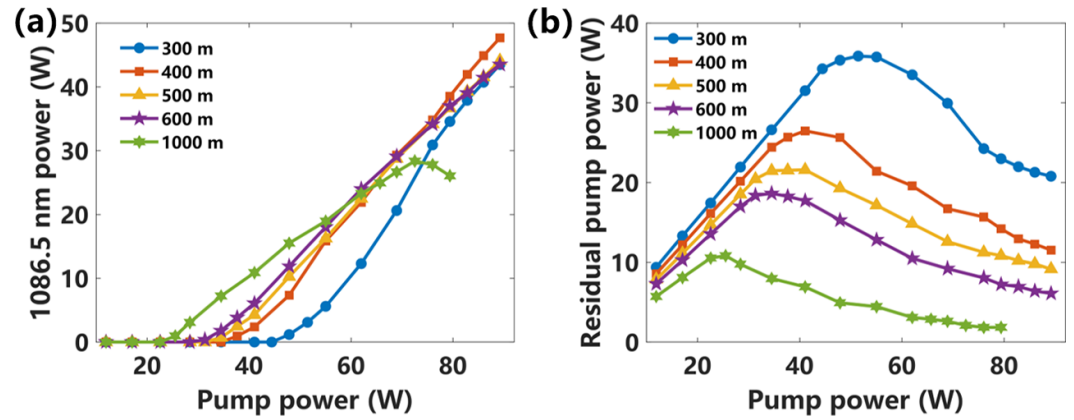

Figure 5. Power evolutions of (a) the $1086.5 \mathrm{~nm}$ signal and (b) the residual pump as functions of pump power, with different PDF lengths.
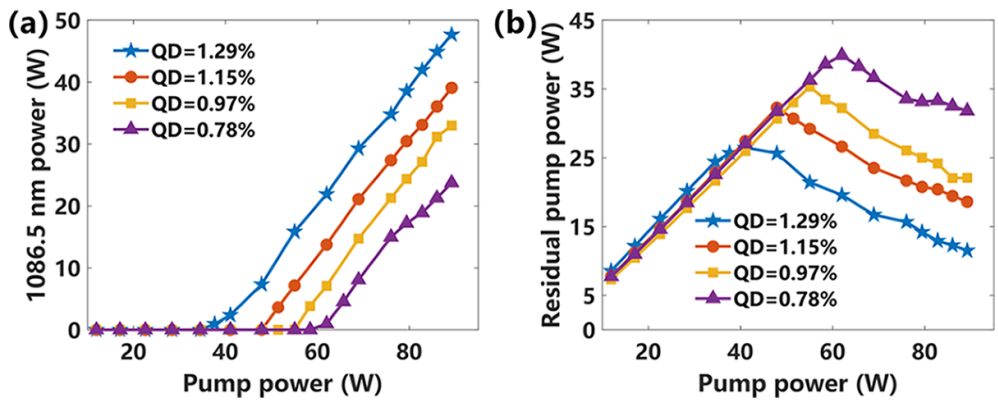

Figure 6. Power evolutions of (a) the $1086.5 \mathrm{~nm}$ signal and (b) the residual pump as functions of pump power, with different QD outputs.

and converted more completely, which results in the lower laser threshold of $1086.5 \mathrm{~nm}$. With $400 \mathrm{~m}$ PDF, the residual pump power is $11.5 \mathrm{~W}$ under the maximal pump power.

To explore the potential on lower QD output (e.g., QD $<1 \%$ ) of the cladding-pumped phosphorus-doped RFL, we tuned the pump wavelength of the ASE source from $1072.5 \mathrm{~nm}$ to 1074,1076 and $1078 \mathrm{~nm}$, with corresponding QDs of $1.29 \%, 1.15 \%, 0.97 \%$ and $0.78 \%$, respectively. Figure 6 describes the power evolutions of the $1086.5 \mathrm{~nm}$ signal laser and the residual pump dependence on different QD outputs, with $400 \mathrm{~m}$ PDF and 19\% OC reflectivity. It can be seen from Figure 6(a) that as the QD is reduced, the generation threshold is higher and the signal power is lower, due to the decreasing Raman gain with smaller frequency shift in the PDF. With a QD of $0.78 \%$, the Raman power rises quickly and achieves $23.7 \mathrm{~W}$ with the maximal pump power of $89.2 \mathrm{~W}$. The corresponding slope efficiency is $77.0 \%$. The increasing generation threshold can be addressed by further optimizations of system parameters, such as OC reflectivity. The low Raman gain provided by the boson peak at a red-shift pump wavelength also causes incomplete pump conversion, as shown in Figure 6(b). After the signal is generated, it can be observed that with the same pump power, the residual pump power is higher with lower QD output. Besides, the low power density of the signal, which is attributed to the weak reflectivity of the OC and the gain fiber with inapposite length, makes a contribution to the insufficient pump absorption. Limited by the wavelength tunable range of the ASE source, the Raman signal with a further reduced QD cannot be investigated in this experiment.
Based on the comprehensive analysis, under the optimal parameters including OC reflectivity of $19 \%$ and PDF length of $400 \mathrm{~m}$, with $1.29 \% \mathrm{QD}$, the maximal signal power achieves $47.7 \mathrm{~W}$ with a slope efficiency of $87.2 \%$, and with $0.78 \% \mathrm{QD}$, the maximal signal power is $23.7 \mathrm{~W}$ with a slope efficiency of $77.0 \%$. The results affirm that the cladding-pumped RFL based on the boson peak of PDF has great ability on low heat production, which may improve the potential on power scaling and thermal management of RFLs.

\section{Conclusion}

In summary, based on the boson-peak-induced Raman gain in PDF, we demonstrate the first cladding-pumped RFL with QD reduced to less than $1 \%$. Compared with core-pumped RFLs, the cladding-pumped RFL has higher maximal signal power and larger residual pump power. The practicability and potential for the high-power and low-QD RFL are experimentally assessed and proved. In future work, we will continue to investigate the properties and improve the performances of phosphorus-doped high-power and low-QD RFLs.

\section{Acknowledgments}

The authors are grateful to Tao Wang, Yanxing Ma, Sen Guo, Weide Hong, Song Zhang and Huan Yang for their important help with this work. This work was funded by the National Natural Science Foundation of China (NSFC) (No. 61905284), the National Postdoctoral Program for Innovative 
Talents (No. BX20190063) and the Innovation Group of Hunan Province, China (No. 2019JJ10005).

Part of the results in this paper were presented at the Advanced Solid State Lasers Conference (ASSL, 2021).

\section{References}

1. Y. Kawahito, H. Wang, S. Katayama, and D. Sumimori, Opt. Lett. 43, 4667 (2018).

2. M. N. Zervas and C. A. Codemard, IEEE J. Sel. Top. Quantum Electron. 20, 219 (2014).

3. W. Shi, Q. Fang, X. Zhu, R. A. Norwood, and N. Peyghambarian, Appl. Opt. 53, 6554 (2014).

4. J. Fan, J. Zhao, L. Shi, N. Xiao, and M. Hu, Adv. Photonics 2, 045001 (2020).

5. Y. Jeong, J. K. Sahu, D. N. Payne, and J. Nilsson, Opt. Express 12, 6088 (2004).

6. T. Matniyaz, F. Kong, M. T. Kalichevsky-Dong, and L. Dong, Opt. Lett. 45, 2910 (2020).

7. S. D. Jackson, Nat. Photonics 6, 423 (2012).

8. C. Jauregui, J. Limpert, and A. Tünnermann, Nat. Photonics 7, 861 (2013).

9. L. Huang, W. Lai, P. Ma, J. Wang, R. Su, Y. Ma, C. Li, D. Zhi, and P. Zhou, Opt. Lett. 45, 4001 (2020).

10. Y. O. Aydin, V. Fortin, F. Maes, F. Jobin, S. D. Jackson, R. Vallée, and M. Bernier, Optica 4, 235 (2017).

11. Y. Fan, B. He, J. Zhou, J. Zheng, H. Liu, Y. Wei, J. Dong, and Q. Lou, Opt. Express 19, 15162 (2011).

12. J. M. Daniel, N. Simakov, A. Hemming, W. A. Clarkson, and J. Haub, Opt. Express 24, 18592 (2016).

13. W. Ke, X. Wang, X. Bao, and X. Shu, Opt. Express 21, 14272 (2013).

14. M. N. Zervas, Opt. Express 27, 19019 (2019).

15. A. V. Smith and J. J. Smith, Opt. Express 19, 10180 (2011).

16. C. Jauregui, C. Stihler, and J. Limpert, Adv. Opt. Photonics 12, 429 (2020).

17. J. Knall, M. Engholm, T. Boilard, M. Bernier, P.-B. Vigneron, N. Yu, P. Dragic, J. Ballato, and M. Digonnet, Optica 8, 830 (2021).

18. J. Knall, M. Engholm, T. Boilard, M. Bernier, and M. J. Digonnet, Phys. Rev. Lett. 127, 013903 (2021).

19. N. Yu, M. Cavillon, C. Kucera, T. W. Hawkins, J. Ballato, and P. Dragic, Opt. Lett. 43, 3096 (2018).

20. T. Yao, J. Ji, and J. Nilsson, J. Lightw. Technol. 32, 429 (2014).

21. S. R. Bowman, Opt. Eng. 56, 011104 (2016).

22. X. Wang, P. Yan, Z. Wang, Y. Huang, J. Tian, D. Li, and Q. Xiao, in Conference on Lasers and Electro-Optics (IEEE, 2018), p. 1.

23. J. Xu, J. Ye, H. Xiao, J. Leng, W. Liu, and P. Zhou, High Power Laser Sci. Eng. 6, e46 (2018).

24. V. R. Supradeepa, Y. Feng, and J. W. Nicholson, J. Opt. 19, 023001 (2017).

25. S. A. Babin, High Power Laser Sci. Eng. 7, e15 (2019).

26. L. Zhang, H. Jiang, S. Cui, J. Hu, and Y. Feng, Laser Photon. Rev. 8, 889 (2014).

27. Y. Shamir, Y. Glick, M. Aviel, A. Attias, and S. Pearl, Opt. Lett. 43, 711 (2018).

28. Y. Feng, L. R. Taylor, and D. B. Calia, Opt. Express 17, 23678 (2009).
29. M. Rekas, O. Schmidt, H. Zimer, T. Schreiber, R. Eberhardt, and A. Tünnermann, Appl. Phys. B 107, 711 (2012).

30. C. Fan, Y. Chen, T. Yao, H. Xiao, J. Xu, J. Leng, P. Zhou, A. A. Wolf, I. N. Nemov, A. G. Kuznetsov, S. I. Kablukov, and S. A. Babin, Opt. Express 29, 19441 (2021).

31. V. R. Supradeepa and J. W. Nicholson, Opt. Lett. 38, 2538 (2013).

32. Y. Chen, T. Yao, H. Xiao, J. Leng, and P. Zhou, High Power Laser Sci. Eng. 8, e33 (2020).

33. Y. Chen, T. Yao, H. Xiao, J. Leng, and P. Zhou, J. Lightw. Technol. 39, 1785 (2021).

34. S. Naderi, I. Dajani, J. Grosek, and T. Madden, Opt. Express 24, 16550 (2016).

35. V. Distler, F. Möller, M. Strecker, G. Palma-Vega, T. Walbaum, and T. Schreiber, Opt. Express 28, 22819 (2020).

36. V. Distler, F. Möller, B. Yildiz, M. Plötner, C. Jauregui, T. Walbaum, and T. Schreiber, Opt. Express 29, 16175 (2021).

37. J. Song, P. Ma, S. Ren, S. Zhang, W. Liu, H. Xiao, H. Zhang, and P. Zhou, Opt. Lett. 46, 2404 (2021).

38. Y. Zhang, J. Xu, J. Ye, J. Song, T. Yao, and P. Zhou, Photon. Res. 8, 1155 (2020).

39. X. Ma, J. Ye, Y. Zhang, J. Xu, L. Huang, J. Leng, Z. Pan, and P. Zhou, Opt. Lett. 46, 2662 (2021).

40. E. Snitzer, H. Po, F. Hakimi, R. Tumminelli, and B. C. McCollum, in Optical Fiber Sensors (OSA, 1988), paper PD5.

41. V. Dominic, S. MacCormack, R. Waarts, S. Sanders, S. Bicknese, R. Doble, E. Wolak, P. S. Yeh, and E. Zucker, in Conference on Lasers and Electro-Optics (OSA, 1999), paper CPD11.

42. H. Lin, Y. Feng, Y. Feng, P. Barua, J. K. Sahu, and J. Nilsson, Opt. Lett. 43, 3080 (2018).

43. A. Hemming, N. Simakov, A. Davidson, S. Bennetts, M. Hughes, N. Carmody, P. Davies, L. Corena, D. Stepanov, and J. Haub, in CLEO: Science and Innovations (OSA, 2013), paper CW1M.1.

44. P. Zhou, L. Huang, J. Leng, H. Xiao, J. Xu, and T. Yao, Sci. Sin. Tech. 50, 123 (2020).

45. J. Nilsson, J. Sahu, J. Jang, R. Selvas, D. Hanna, and A. Grudinin, in Optical Amplifiers and Their Applications (OSA, 2002), paper PD2.

46. C. A. Codemard, J. Ji, J. K. Sahu, and J. Nilsson, Proc. SPIE 7580, $75801 \mathrm{~N}(2010)$.

47. Y. Glick, Y. Shamir, M. Aviel, Y. Sintov, S. Goldring, N. Shafir, and S. Pearl, Opt. Lett. 43, 4755 (2018).

48. Y. Chen, T. Yao, H. Xiao, J. Leng, and P. Zhou, Opt. Lett. 45, 2367 (2020).

49. J. Ye, J. Xu, Y. Zhang, J. Song, J. Leng, and P. Zhou, J. Lightw. Technol. 37, 3113 (2019).

50. Y. Chen, J. Song, J. Ye, T. Yao, J. Xu, H. Xiao, J. Leng, and P. Zhou, Opt. Express 28, 12395 (2020).

51. X. Cheng, W. Pan, X. Zeng, J. Dong, S. Cui, and Y. Feng, Opt. Fiber Technol. 54, 102119 (2020).

52. J. Ye, J. Xu, J. Song, Y. Zhang, H. Zhang, H. Xiao, J. Leng, and P. Zhou, Photon. Res. 7, 977 (2019).

53. A. Liu and K. Ueda, Opt. Commun. 132, 511 (1996).

54. H. Jiang, L. Zhang, and Y. Feng, Opt. Express 23, 13947 (2015).

55. S. Wang, S, "Raman assisted four-wave mixing in optical fiber and its applications," $\mathrm{PhD}$. Thesis (The Hong Kong Polytechnic University, China, 2010). 